\title{
FREGEAN INCOMPLETENESS
}

\author{
EDWIN MARTIN, JR.
}

The Fregean theory of syntax says what the meaningful parts of sentences are, and which combinations of those parts are meaningful. The Fregean theory of meaning says how the meaningful parts of a meaningful expression contribute to that expression's sense and reference. The theory of syntax divides the meaningful parts of sentences into proper names (including sentences) and function names. (See $[3], \S \S 1-13 ;[8], \S 1$ ) Function names are further distinguished according to numbers of argument positions and the syntactic type of expression appropriate to each position. A function name which takes in its argument places proper names is first-level; a function name which takes in its argument places first-level function names is second-level. And so on. ([3], §§21-23.) Every complex meaningful expression is the result of completing a function name with expressions of the appropriate types; and, conversely, every such completion is a complex meaningful expression. $([2], \S 30$.) Since proper names and function names are of different syntactic categories, substitution of one for the other in a meaningful expression will not in general preserve meaningfulness. It is also important to recognize that function names of different types are of different syntactic categories, and therefore substitution of a function name for another of a different type will not preserve meaningfulness. ([3], pp. 73, 77; [4], p. 50.)

Frege thinks of function names as having argument places or positions. These positions are marked by lower case Greek consonants he calls "gap holders". These gap holders serve several purposes. They indicate where the function name is to be completed, where the completing expressions fit. They also specify the pattern of completion. For example,

(1) $\xi$ beat $\xi$ 's record

is to be completed by filling both of its argument positions with the same expression, as in 
EDWIN MARTIN, Jr.

(2) Eddy beat Eddy's record

or in

(3) Jacques beat Jacques's record.

The predicate

(4) $\xi$ beat $\zeta$ 's record,

on the other hand, may be completed by filling the argument places differently, as in

(5) Eddy beat Jacques's record.

Another job the gap holders perform is marking the type of the completing expression, thus displaying the syntactic category of the function name. Frege uses ' $\xi$ ' and ' $\zeta$ ' to mark positions appropriate to proper names, and ' $\varphi$ ' and ' $\psi$ ' for positions appropriate to firstlevel function names. Gap holders are therefore an integral part of the theory of syntax for they both mark where other expressions can be inserted as well as the syntactic category of the function name.

The Fregean theory of meaning assigns objects to proper names as senses and references, and functions to function names. The reference of a complex expression is the value of the function which is the reference of the expression's main function name when it takes as arguments the references of the expressions which fill the main function name's argument places. ([3], p. 34.) Analogously, the sense of a complex expression is a function of the senses of its parts. Sentences have truth values for references and thoughts for senses.

Proper names and sentences are "complete" expressions, according to Frege, and their senses and references - viz., objects - are "complete," "saturated", "not in need of completion". ([3], §2.) The case is otherwise however for predicates, connectives, and quantifiers: they are "incomplete" expressions, and their senses and references are "unsaturated" and "in need of completion". ([3], \&1; [4], pp. 24, 31, 115.) Thus Frege says,

The thought does not, by its make-up, stand in any need of completion; it is self-sufficient. Negation on the other hand needs to be completed by a thought. The two components, if we choose to employ this expression, are quite different in kind and contribute quite differently towards the formation of the whole. $([5]$, p. 49.) 
Many commentators have worried about what such completeness and incompleteness might amount to. Max Black, for instance, has toyed with the idea that a function is an unfinished object ([1]). And William Marshall has sought to dispense with metaphor by saying that functions, "being perforated, bind the other parts together" ([7], p. 255). My purpose is to offer a view of this, one which I hope will take some of the mystery out of Frege's work.

There is, prima facie, a clear, literal sense in which function names can be said to be incomplete: they do, after all, contain gaps which are filled to form proper names. Now Dummett says that for Frege the incompleteness of an expression amounts to the fact that the expression

does not consist merely of some sequence of words or symbols, but in the occurrence within sentences of such a sequence standing in a certain uniform relation to terms occurring in those sentences. ([2], p. 31)

Thus (2) and (3) contain the same predicate (viz., (1)), while (5) contains a different predicate (namely, (4)0. Yet (5) has as much orthographic overlap with (2) and (3) as they have with each other. Dummett's point is that what predicate is involved depends not just on what words it contains, but also on the pattern of words filling its argument places; and "it is precisely in this sense," he claims, "that an expression is said by Frege to be 'incomplete'."

Although there seems to be some truth in what Dummett says here, it does not appear that this could be Frege's whole point in calling a predicate "incomplete". For as Dummett himself notes, since for predicates with one argument position there is no question of the pattern of filling argument places, Frege should not - on Dummett's account - think of them as incomplete. Further, instead of using Greek consonants to mark the argument positions in a predicate, we might use indexed sets of parentheses. Thus we could say that (2) and (3) contain the predicate

$$
\text { ( ) beat ( ) 's record }
$$

while (5) contains the different predicate

$$
\text { ( ) beat ( })_{2} \text { 's record. }
$$

We could then leave in the parentheses in sentences - recasting (5) as '(Eddy) $)_{1}$ beat (Jacques) $)_{2}$ 's record' - thereby exhibiting the pattern of completion of the predicate. If we did this, then the predicate 
would indeed "consist merely of some sequence of words or symbols" for now the gap holders would literally be present, even when the gaps were filled. Such a change, it seems, would be a minor, orthographic modification, and, one would think, nothing important to Frege's thought would be altered. But if Dummett is right, instituting this change would take from Frege every reason he had to think of predicates as "incomplete"; the incompleteness of predicates, that is, is on Dummett's view an orthographic accident.

Such conclusions, I think, are farfetched. Even if he had used indexed parentheses for gap holders, it seems clear that Frege would have maintained the incompleteness of predicates. And attributions of incompleteness to one-place predicates by Frege is not mere sloppiness or laziness. Much of the point Frege is making here is the difference of predicates from proper names. They are of different syntactic categories, and this Frege emphasizes in terms of completeness. Analogously, a function name with two argument positions is said to be "doubly incomplete", thus emphasizing its syntactic difference from function names with only one argument position. And the incompletenesses of function names of different levels will be seen as different too. So talk about the incompleteness of expressions is a way of talking about syntactic categories.

Frege believed that the thought expressed by a sentence is composed of parts which are analogs to the syntactic division of the sentence into parts (cf. [5], p. 38). That is, corresponding to the division of words into syntactic categories, Frege envisions a division of senses (and references) into types. This parallel is necessary if the theory of syntax is to be consistent with the theories of sense and reference (cf. [8], §2). Consequently the distinctions of semantic categories are as important to the theory of meaning as the distinctions of syntactic categories are to the syntax.

Now just as Frege talks of the incompleteness of expressions to separate them into syntactic categories, so too he talks of the unsaturatedness of functions to separate them into types. And he must make this second separation if his theories of syntax and meaning are to fit together. This is the principal goal of Frege's use of "incomplete" and "unsaturated": they provide a way of talking about categorial differences. Why, though, did Frege fix on the terminology he uses? His choice of language was probably influenced by what was for him the newly promising chemical theory, with its positive and negative radicals being "saturated" to form complete compounds. (Compare [4], p. X, note 2.) İn an early unpublished manu- 
script Frege makes this link, saying that functions and objects, as well as their names, are like

the behavior of the atom: we suppose an atom never to be found on its own, but only combined with others, moving out of one combination only in order to enter immediately into another. A sign for a property never appears without a thing to which it might belong being at least indicated, a designation of a relation never without indication of the things which might stand in it. $([6]$, p. 17.)

It seems clear that Peirce, apparently independently and almost simultaneously, used the same language as Frege under just this influence. Speaking of relation-words ("relatives"), Peirce says,

A chemical atom is quite like a relative in having a definite number of loose ends or "unsaturated bonds", corresponding to the blanks of the relative. In a chemical molecule, each loose end of one atom is joined to a loose end, which it is assumed must belong to some other atom ... Thus the chemical molecule is ... like a complete proposition. ([9] , 3.469; see too $3.421,3.475$ )

This is obviously a very enticing analogy. (Peirce notes that the analogy quickly breaks down. "Yet," he says, "I cannot resist the temptation to pursue it.") For in assigning various "valencies" to chemical objects, the possibilities of lawful combination are defined: the objects are assigned types, or categories, and chemical laws are stated that specify, in terms of the categories, which combinations are possible. This is precisely the goal of a theory of syntax, and of a theory of meaning; only the objects being sorted out here are not chemical, but are syntactic or semantic.

It may be said with some force that there is nothing inherently "incomplete" about a positive or negative radical. It is incomplete in just the sense that it is a proper part of larger molecules, and it is these molecules which are the naturally occuring objects, not the radical; so, in its natural state the radical is a proper part of a larger compound. Much the same things are said by Frege for a function name, and for its sense. There need not be any inherent incomplete. ness with these: they are not unfinished objects. They are incomplete precisely in the sense that in their "natural states" they are proper parts of bigger compounds. Here we need to think of the naturally occurring objects as being sentential in nature: sentences, truth 
values; thoughts. Frege is forced by what he sees as the syntactic parity of sentences and proper names into extending this a bit; thus we will really need to think of the naturally occurring objects as being not just sentential, but as either sentential or nominal. This extension is perhaps a place where the analogy begins to look thin, for proper names (or their senses) hardly seem to have as good a claim to natural occurrence as do sentences (or thoughts). But, in any event, the main point of the analogy remains the assignment of the meaningful entities of the theories of syntax and sense into systems of non-overlapping categories, and the stating of laws that specify which combinations of which categories are possible.

The chemical parallel is instructive. If all chemical compounds were formed by ionic bonds between charged particles, then it would be true that neutral atoms could not enter into combination with one another. Positive and negative radicals would be necessary for forming compounds and, in a picturesque moment, we might put this by saying that they were the glue of the compound, what held it together. But it is clear that there would be no point in trying to find some deep metaphysical sense to this; it is just a flamboyant way of describing the combinatorial facts. Similarly, every sentence must contain a function name, and this combinatorial fact might be put by saying that function names provide the glue for compound expressions. If we put the fact this way - as Frege does - we are being picturesque, not deeply metaphysical.

Talk of degrees and kinds of incompleteness, then, is a way of delineating syntactic and semantic categories in preparation for the statement of syntactic and semantic laws of meaningful combination in terms of those categories. It is a picturesque way of describing the situation suggested by the chemical parallel and the inclination to think of function names as containing holes. But it is mistaken to look behind the talk, past the combinatorial laws, for something that looks like glue or is what the function becomes when finished.

\section{INDIANA UNIVERSTTY BLOOMINGTON, INDIANA 47401}

USA

\section{REFERENCES}

[1] Max Black, "Frege on Functions", reprinted in Essays on Frege (Edited by E.D. Klemke; Urbana, University of Illinois Press, 1968). 


\section{FREGEAN INCOMPLETENESS}

[2] Michael Dummett, Frege: Philosophy of Language (New York, Harper \& Row, 1973).

[3] Gottlob Frege, The Basic Laws of Arithmetic (Edited and translated by Montgomery Furth; Berkeley, University of California Press, 1964).

[4] Gottlob Frege, Translations from the Philosophical Writings (Edited and translated by P.T. Geach and Max Black; Oxford, Blackwell, 1966).

[5] Gottlob Frege, Logical Investigations (Edited and translated by P.T. Geach and R.H. Stoothoff; New Haven, Yale University Press, 1977).

[6] Gottlob Frege, Posthumous Writings (Edited by Hermes, Kambartel, and Kaubach; University of Chicago Press, Chicago, 1979).

[7] William Marshall, "Frege's Theory of Functions and Objects", reprinted in Essays on Frege (Edited by E.D. Klemke; Urbana, University of Illinois Press, 1968).

[8] Edwin Martin, "Frege's Problems with "The Concept Horse" ", Critica, Vol. V, No. 15 (1971), 45-61.

[9] Charles Sanders Peirce, The Collected Papers of Charles Sanders Peirce, Vol. 3 (Edited by Charles Hartshorne and Paul Weiss; Cambridge, Harvard University Press, 1931). References are to paragraph numbers. 